\title{
Article
}

\section{Initial Validation of the Suicide Competency Assessment Form among Behavioral Health Staff in the National Health Services (NHS) Trust. Archives of Suicide Research}

Cramer, Robert J, Ireland, Jane Louise, Long, MM, Hartley, Victoria and Lamis, DA

Available at http://clok.uclan.ac.uk/25943/

Cramer, Robert J, Ireland, Jane Louise ORCID: 0000-0002-5117-5930, Long, MM, Hartley, Victoria and Lamis, DA (2019) Initial Validation of the Suicide Competency Assessment Form among Behavioral Health Staff in the National Health Services (NHS) Trust. Archives of Suicide Research. Archives of Suicide Research . ISSN 1381-1118

It is advisable to refer to the publisher's version if you intend to cite from the work. http://dx.doi.org/10.1080/13811118.2019.1577194

For more information about UCLan's research in this area go to http://www.uclan.ac.uk/researchgroups/ and search for <name of research Group>.

For information about Research generally at UCLan please go to http://www.uclan.ac.uk/research/

All outputs in CLoK are protected by Intellectual Property Rights law, including Copyright law. Copyright, IPR and Moral Rights for the works on this site are retained by the individual authors and/or other copyright owners. Terms and conditions for use of this material are defined in the policies page. 
Initial Validation of the Suicide Competency Assessment Form among

Behavioral Health Staff in the National Health Services (NHS) Trust

Cramer, R.J., Ireland, J.L., Long, M.M., Hartley, V., \& Lamis, D.A. (In Press). Initial Validation of the Suicide Competency Assessment Form among Behavioral Health Staff in the National Health Services (NHS) Trust. Archives of Suicide Research. 


\begin{abstract}
Objective: The Suicide Competency Assessment Form (SCAF) provides a framework for suicide prevention skills training. This study assessed SCAF psychometric properties in a sample of behavioral health staff. Method: A cross-sectional survey of National Health Services (NHS) staff from varying disciplines $(N=170)$ was conducted. Results: The SCAF yielded a one-factor structure with high internal consistency. Nursing assistants reported lower SCAF scores compared to other professionals. SCAF scores demonstrated positive associations with prior suicide prevention training, job enthusiasm, and several suicide/self-injury prevention outcome expectations (i.e., optimism working with self-harming patients and perceived ability to help self-harming patients). SCAF scores further demonstrated incremental validity in the form of multivariate model associations with suicide/self-injury prevention outcome expectations. Improved job satisfaction mediated the pathway from SCAF scores to perceived ability to help self-harming patients. Conclusion: The SCAF can be utilized in suicide prevention training and clinical supervision.
\end{abstract}

Key Words: Suicide; Competency; Staff; Burnout; Job Satisfaction; National Health Services 
Suicide prevention is a worldwide public health priority that warrants considerable assessment and intervention (WHO, 2015). In the United Kingdom (UK), for example, public health statistics suggest almost 6,000 persons die by suicide annually (Samaritans, 2017), yielding a population-based rate of 10.1/100,000 persons (ONS, 2018). Moreover, suicide has far-reaching impacts on family, friends, as well as tremendous economic costs in medical and work loss annually (Cerel et al., 2018; Shepard et al., 2016). A number of strategies to enhance workforce responses to suicide (e.g., gate-keeper and educational trainings) have been implemented and evaluated (e.g., Arensman, 2017; Ferguson et al., 2018). Behavioral health providers, in particular, are in a unique position to assess suicide and make potentially life-saving decisions about suicide risk management (Roush et al., 2018). The present study, therefore, seeks to validate an assessment instrument of behavioral health staff suicide prevention competencies in order to provide structure for future evidence-based suicide prevention training.

\section{Behavioral Health Provider Suicide Prevention Contacts, Competencies and Training}

Studies (e.g., Ahmedani et al., 2014; Luoma et al., 2002) have found that approximately $20 \%$ of individuals who die by suicide have had contact with a behavioral health professional within one month prior to their death. Consequently, educating behavioral health professionals is critical for effectively intervening and preventing suicide (Silva et al., 2016). However, training in the assessment and management of suicide risk has been inadequate for this group of providers (Schmitz et al., 2012). Speaking to this problem, a recent study (Awenat et al., 2017) of inpatient and outpatient National Health Services (NHS) behavioral health staff in Northern England showed that consequences of experiencing patient suicide-related behavior (e.g., fear of being blamed) in combination with negative views of suicide (e.g., as inevitable and untreatable) yielded fear and resistance for staff to engage in future prevention efforts. Awenat and 
colleagues concluded that suicide prevention training and support for staff need to go beyond risk assessment strategies to include clinical service delivery and other skills. A dense literature echoes consensus that an empirically-based theory-driven approach for behavioral health providers in the assessment and treatment of suicidal behaviors is of upmost importance (Jahn et al., 2016; Mitchell et al., 2017; Roush et al., 2018). As such, the present investigation offers some of the first psychometric development of a tool encompassing clinical care skills (e.g., intervention) and provider attitudes/reactions. As outlined below, this instrument can assess provider self-perceived suicide prevention skills while also serving the purposes of helping clinical supervision and self-reflective practice.

Suicide prevention competencies commonly address knowledge and attitudes (e.g., Batterham et al., 2013; Botega et al., 2005, 2007). Existing evidence suggests that suicide and self-harm related attitudes span issues concerning morality of suicide, positive/negative views about suicidal patients or persons who die by suicide, and beliefs about the clinician's own ability to help patients experiencing suicide and self-harm (e.g., Batterham et al., 2013; Botega et al. 2005, 2007). Researchers have found that behavioral health professionals' attitudes about self-harm and suicide may influence their willingness and ability to identify, assess, and treat high-risk patients (Jiao et al., 2014; Long, Manktelow, \& Tracey, 2013). Thus, changing negative attitudes among providers who are likely to encounter individuals at increased risk for suiciderelated behavior suggests a promising intervention point. Other impacts of provider experiences with and attitudes toward patient suicide concern job satisfaction and burnout, particularly when working with suicidal patients (Fleury et al., 2017; Morse et al., 2012). It is important for behavioral health professionals who encounter suicidal patients to practice self-care and employ strategies to prevent burnout and exhaustion (Brems \& Johnson, 2009). 


\section{The Suicide Competency Assessment Form: Conceptualization and Development}

In a step toward consistency, Cramer and colleagues (2013, 2016, 2017) proposed a set of 10 suicide prevention core competencies to frame training for behavioral health providers. Based on a review of established suicide prevention competencies in the literature (e.g., Kleespies \& Bongar, 2009; Rudd, 2006), the Suicide Competency Assessment Form (SCAF; Cramer et al., 2013; see electronic supplementary material [ESM] Appendix for list of competencies), a training tool was developed for evaluating self- and observer-rated skills in each domain. Four studies to date have used the SCAF as an outcome for training effectiveness based on coverage of the 10 core competencies. Studies featured (1) in-person workshop for community mental health and counseling center professionals, and psychology postgraduate trainees (Cramer et al., 2017; La Guardia et al., in press); (2) in-person semester-long postgraduate course for clinical psychology students (Cramer et al., 2016); (3) online semester-long course for an interprofessional group of health professions students (Cramer \& Long, in press). The latter study adapted the SCAF to include public health content and skill mastery. Across studies, the SCAF has been used as a single-factor total score. Internal consistency values have been high (.94-.96; La Guardia et al., in press). Correlational and between-groups analyses with the SCAF are largely lacking to date. However, the total and global scores have demonstrated meaningfully sized positive changes in response to the range of training formats (Cramer et al., 2016, 2017; Cramer \& Long, in press; La Guardia et al., in press).

\section{The Present Study: SCAF Factor Structure, Reliability and Validity}

The present study addresses several gaps in the suicide prevention training literature.

First, providers suicide-related attitudes and beliefs pertaining to effective suicide prevention skills can impact patient outcomes (Awenat et al., 2017), yet no measure to date captures 
assessment of these competencies. Specific to SCAF development, no work exists regarding factor structure. Although prior suicide attitude measures often contain general subscales of professional capacity (e.g., Botega et al., 2005; Gibb et al., 2010), item content to date does not cover a range of perceived skills. There is reason to consider a single versus bi-factor structure given that suicide prevention competencies include both provider (e.g., self-care; Brems \& Johnson, 2009) and practice delivery (e.g., enacting evidence-based treatment plans; Rudd, 2006) skills. Beyond previous utility in staff needs assessment and as a training outcomes, examining the factor structure may enhance patient care through use of the SCAF as a structured method of training and supervision of suicide prevention practitioners. Use of the SCAF as an evidencebased framework fits well within healthcare systems (e.g., Zero Suicide; Brodsky et al., 2018). For instance, the SCAF clinical care competencies provide structure for training and application of evidence-based suicide prevention assessment and intervention strategies. Moreover, the SCAF fits well within asocial-ecological conceptualization of suicide risk and prevention (Cramer \& Kapusta, 2017) in that the measure includes individual level (e.g., eliciting risk and protective factors for an individual patient; establishing an evidence-based intervention plan) and interpersonal level (e.g., addressing provider attitudes as medical determinants of health outcomes; involving social support in prevention planning) approaches to suicide prevention.

The present investigation also builds on the limited SCAF construct validity to date by examining demographic, experiential, and work-related correlates. The broader suicide prevention attitudes literature can shed some light on potential demographic, experiential and work-related associations of the SCAF. Research concerning the associations of age, and experience is mixed (Gibb et al., 2018; Botega et al, 2005). Regarding staff profession, a systematic review (Saunders et al., 2012) demonstrated that doctors hold more negative attitudes 
compared to nurses. Moreover, among nursing professionals, nursing assistants reported highest levels of professional capacity to work with suicidal patients (Botega et al., 2005).

Negative attitudes toward patients engaging in suicide-related behavior has been associated with a range of job-related outcomes such as burnout (i.e., psychological exhaustion; low personal accomplishment; Gibb et al. 2010) and hesitancy to talk about suicide with patients (Awenat at el., 2017). The SCAF assesses perceived abilities or mastery of suicide prevention competencies, a construct that differs from general attitudes toward suicide/self-harm. Even where professional capacity or abilities are measured, assessment tools do not assess a full range of skills. While we rely on the broader suicide-related attitudes literature as a foundation for construct validity, this is the first study to examine in-depth correlates of a comprehensive tool assessing perceived suicide prevention abilities. Doing so is important for behavioral healthcare settings in part because suicide prevention abilities may represent a modifiable skill set that may impact a broader range of workforce health and retention outcomes. Where perceived suicide prevention skills demonstrate convergent with attitudes toward suicidal/self-harming patients and related prevention and intervention outcomes, we conduct: (a) regression models to assess SCAF incremental validity associations with job-related and beliefs about patient suicide/self-harm (controlling for demographics), and (b) mediation tests to assess whether perceived skills may be associated with expectations about prevention efforts and patient outcomes. Social-cognitive theories of health behavior (e.g., Bandura, 1997, 2004) and suicide prevention training (e.g.. Burnette et al.., 2015) support such analytic questions. For instance, Bandura's (2004) selfefficacy approach to health education and training suggests that specific skill mastery should be associated with more positive perceptions of outcome expectations for those skills. Applied to the present context, as the SCAF is an assessment of specific suicide prevention skills (Cramer et 
al., 2013), higher self-perceived scores should be associated with positive perceptions of working with patients experiencing suicidality/self-harm, as well as expected outcomes of intervention and prevention efforts. As such, SCAF scores represent more than a similar attitudinal construct as prior suicide and suicide prevention attitudes scales; in assessing specific prevention skills for the first time, we can test theoretically-based incremental validity and mediation pathways to suicide prevention-related expectations about patient well-being outcomes, a noteworthy domain linked with actual patient care efforts and well-being (Awenat et al., 2017).

We hypothesized:

H1: The SCAF will demonstrate a single-factor structure.

H2: The SCAF total score will demonstrate high internal consistency.

H3: Nursing staff will demonstrate the highest perceived suicide prevention competency scores. We explore additional SCAF associations with age and professional experience.

H4: SCAF scores will demonstrate negative associations with burnout.

H5: SCAF scores will demonstrate positive associations with job enthusiasm and job satisfaction.

H6: SCAF scores will be associated with positive suicide and self-injury related attitudes. H7: Controlling for demographic and experiential factors, SCAF scores will be positively associated with better job-related outcomes (i.e., lower burnout, higher enthusiasm, and higher satisfaction).

H8: Controlling for demographic and experiential factors, SCAF scores will be positively associated with attitudes and expectations regarding patient suicide and self-injury prevention and treatment. 
Exploratory question: In light with social-cognitive perspectives summarized, we will also conduct follow-up mediation tests to explore pathways from SCAF scores to suicide and selfinjury related attitudes.

\section{Method}

\section{Participants}

Healthcare professionals $(N=170)$ across three NHS Trust secure psychiatric hospitals were included in the study. Participants were drawn from one low, one medium, and one high secure facility housing adult male psychiatric patients. For international context, NHS secure facilities house all in-patient populations, with self-harm or suicide among the required or most common admission criteria for low secure admission and above (Crichton, 2009). Security level is differentiated by factors such as the nature of patient violence, stability and insight, as well as treatment staff/setting capacity to respond (Crichton, 2009). ESM Table 1 provides primary demographic characteristics and descriptive statistics for the sample. Staff included a slight majority female sex, middle adult age, considerable amount of experience (i.e., years of clinical experience, working in current secure facility), a modest amount of prior suicide prevention training, mostly nursing assistants or psychiatric nurses, majority of who had never lost a patient to suicide and most staff knowing someone who died by suicide.

\section{Measures}

Demographics. Participants completed a self-report survey of background information, including: sex, discipline (e.g., psychologist, nursing assistant), age, experience with suicide (i.e., loss of patient to suicide, known someone to die by suicide), and professional training (i.e., hours of previous suicide prevention training, years of clinical experience, years in inpatient facility). 
Suicide Competency Assessment Form (SCAF; Cramer et al., 2013). The SCAF contains an item for each of the 10 core competencies (see literature review and ESM Appendix) and an overall rating of competence. Each of the ten competencies is rated on the following scale: 1 ("incapable"), 2 (“working towards competency"), 3 (“competent"), or 4 (“advanced"). These 10 items can be summed for a total score. Also, a global rating of competency is rated on the following 8-point scale: "unacceptable" (1-2), "working towards competency" (3-4), “competent" (5-6), "advanced" (7-8).

Job-related outcomes. Burnout was assessed with the English version of the Spanish Burnout Inventory (SBI; Gil-Monte \& \& Olivares, 2011; Gil-Monte \& Manzano-Garcia, 2015). Having been validated cross-culturally, the SBI contains 20 items, each rated on a five-point scale (0-4). Example psychometric properties show the measure contains four subscales of job burnout among professionals: (1) Enthusiasm toward job ( $\alpha=.87$, (2) Psychological Exhaustion ( $\alpha=.86$ ), (3) Indolence ( $\alpha=.76$ ), and (4) Guilt ( $\alpha=.80)$ (Gil-Monte \& Manzano-Garcia, 2015). The present study applies the SBI in an English-speaking sample, with resulting internal consistencies for the present study of: Enthusiasm toward job ( $\alpha=.87)$, Psychological Exhaustion $(\alpha=.82)$, Indolence $(\alpha=.73)$, and Guilt $(\alpha=.65)$.

Participants completed a ten-question forensic mental health job-specific satisfaction survey developed in consultation with NHS research staff (including co-author JL). Each item was rated on a 5-point scale with higher scores reflecting greater agreement. Topics covered included level of satisfaction with: (1) overall working conditions, (2) management/leadership, (3) time for self-reflective practice, (4) content of self-reflective practice, (5) derivation of meaning from working with patients, (6) provision of continuing education/training, (7) fear of 
injury, (8) fear of patient self-directed violence, (9) experience of negative feelings on the job, and (10) overall job satisfaction. A summed total score was used in the present study $(\alpha=.83)$.

Suicide and self-injury prevention attitudes. Self-injury prevention related attitudes were assessed with the Attitudes toward Self-Harm Patients (ASHP; Gibb et al., 2010). The ASHP contains 15 items, each rated on a four-point scale with higher scores denoting more positive attitudes. Several items are reverse-scored. Three subscales are tabulated: (1) perceived ability to effectively help patients, (2) optimism and patience, and (3) confidence and adequacy of training. Internal consistencies were not reported in the development article. Internal consistency values in the present study were: perceived ability to effectively help patients $(\alpha=.65)$, optimism and patience ( $\alpha=.61)$, and confidence and adequacy of training $(\alpha=.64)$.

Suicide prevention-related attitudes were measured with the Attitudes to Suicide Prevention Scale (ASP; Herron et al., 2001). The ASP is a 14-item $(\alpha=.77)$ instrument with each statement rated on a 5-point scale ranging from 5 (strongly disagree) to 1 (strongly agree). One item is reverse-scored. All items are summed for a total score where higher scores reflect negative attitudes. Internal consistency was acceptable in the present sample $(\alpha=.74)$.

\section{Procedure}

Behavioral health staff were recruited via notification of a voluntary research survey advertised via posters and verbal recruitment at all three secure facilities. A member of the research team $(\mathrm{VH})$ visited each site and provided a verbal summary of the benefits and procedure of the study. Interested staff were given a written explanation of the purposes. Consent was obtained prior to the administration of the survey and participants were debriefed upon study completion. Data collection occurred in both online and paper-pencil formats. ${ }^{1}$ The study

\footnotetext{
${ }^{1}$ Data collection source did not affect SCAF total, $t(168)=-0.30, p=. .766$, or global, $t(168)=0.75, p=.457$, scores
} 
received ethics approval from the University of Central Lancashire and Old Dominion University Human Subjects Review Boards.

\section{Data analysis}

All data analyses were conducted via SPSS v. 24 (see ESM for full data analysis explanations).

\section{Results}

H1 \& H2: SCAF Internal Consistency and Exploratory Factory Analysis. KaiserMeyer-Olkin (KMO) measure of sampling adequacy was ideal $(\mathrm{KMO}=.90)$, indicating the presence of meaningful relationships among the SCAF items. The Bartlett's Test of Sphericity indicates the presence of meaningful correlations among the factors, $\chi^{2}(45)=645.49, p<.001$. Consistent with H2 and supported by visual inspection of the scree plot (see ESM Figure 1), one factor emerged from the 10 -item scale. All 10 items loaded positively on Factor $1(\lambda$ range $=.53$ .78 , eigenvalue $=4.79,47.9 \%$ variance accounted for; see ESM Table 2 for full factor loading results). Confirming H2, the SCAF total score displayed good internal consistency $(\alpha=.88)$. ESM Table 2 demonstrates that the mean SCAF total score is 2.64 (26.38/10 items), which suggests a mean score between "working toward competence" and "competent." The mean SCAF global score of 4.33 suggests a similar level of perceived overall competence. The SCAF total and global scores demonstrated a large significant positive correlation $(r=0.62, p<.001)$.

SCAF Concurrent Validity: Demographics, Job-related Outcomes, and Suicide/Selfinjury Prevention Attitudes. The SCAF total score displayed non-significant associations with: sex, $t(167)=-0.38, p=.702$; losing a patient to suicide, $t(168)=-1.06, p=.292$; and knowing someone who died by suicide, $F(5,163)=0.47, p=.796$. The SCAF total score significantly varied 
by participant discipline, $\mathrm{T}(168)=4.04, p<.001 .^{2}$ Contrary to $\mathrm{H} 3$, nursing assistants $(M=24.38$, $S D=5.64$, Cohen's $d=0.63,95 \% \mathrm{CI}=0.32-0.94)$ reported significantly and moderately sized lower mean SCAF total scores compared to other professionals $(M=27.82, S D=5.33)$.

ESM Table 3 contains correlations between the SCAF total score and construct validation variables. The SCAF total score demonstrated the following significant patterns: (1) small positive associations with job enthusiasm and optimism/patience working with self-harming patients, (2) medium positive associations with previous hours of suicide prevention training, job satisfaction, and perceived ability to help self-harming patients, and; (3) a medium negative association with negative attitudes toward suicide prevention. Regarding the latter finding, this can also be understood as a positive correlation with positive attitudes regarding suicide prevention because higher scores reflect negative attitudes. Therefore, $\mathrm{H} 4$ regarding burnout was supported; whereas, H5 (for job enthusiasm, satisfaction) and H6 (for optimism/patience, perceived capacity to help self-harming patients, and general suicide prevention attitudes) were partially supported.

SCAF incremental validity: Multivariate regression models assessing associations with job-related attitudes and suicide/self-injury attitudes/outcome expectations. Two models were run, one for each set of significant bivariate correlates of SCAF total scores: (1) job-related outcomes (i.e., SBI enthusiasm for the job and overall job satisfaction), and (2) suicide/self-injury prevention attitudes (i.e., negative attitudes toward suicide prevention, perceived ability to help self-harming patients, and optimism/patience working with self-harming patients). The set of predictors in each model included the following control variables: sex

\footnotetext{
${ }^{2}$ Low subgroup cell counts for several sub-disciplines, in combination highlighting nursing assistants as potentially varying in suicide-related attitudes, resulted in the decision to collapse other disciplines in order to examine nursing assistants versus other professionals.
} 
(female coded as reference group), lost patient to suicide (no coded as reference group), discipline (other coded as reference group), known someone who died by suicide (no coded as reference group), age, years of clinical experience, years working in current facility, and hours of suicide prevention training.

ESM Table 4 contains the multivariate statistics for the job-related outcomes model. Contrary to $\mathrm{H} 7$, the SCAF total score displayed a non-significant multivariate effect, precluding further inspection of univariate effects. The only significant and moderate multivariate effects were observed for male sex and knowing someone who died by suicide. Specifically, males, compared to females, reported greater job enthusiasm $\left(\beta=1.79, \operatorname{Se} \beta=0.72, p=.013, \eta_{\mathrm{p}}^{2}=.04,95 \%\right.$ $\mathrm{CI}=.00-.11)$, less indolence $\left(\beta=-1.58, \mathrm{Se} \beta=0.47, p=.001, \mathrm{\eta}_{\mathrm{p}}{ }^{2}=.07,95 \% \mathrm{CI}=.01-.15\right)$, and higher job satisfaction $\left(\beta=2.23 \mathrm{Se} \beta=0.98, p=.025, \mathrm{\eta}_{\mathrm{p}}^{2}=.03,95 \% \mathrm{CI}=.00-.10\right)$ reported less enthusiasm for the job compared to other staff. Those who knew someone who died by suicide also reported higher psychological exhaustion compared to those who did not know someone who died by suicide $\left(\beta=1.75 \mathrm{Se} \beta=0.56, p=.002, \mathrm{\eta}_{\mathrm{p}}^{2}=.06,95 \% \mathrm{CI}=.01-.14\right)$.

ESM Table 5 contains multivariate statistics for the suicide/self-injury attitudes model. The SCAF total score displayed a significant medium multivariate effect, and was the only significant predictor in the model. Specifically, consistent with H8, the SCAF total score displayed: (1) a significant positive and medium sized association with perceived ability to help self-harming patients $\left(\beta=0.92, \operatorname{Se} \beta=0.21, p<.001, \mathrm{\eta}_{\mathrm{p}}^{2}=.11,95 \% \mathrm{CI}=.03-.18\right)$; (2) a significant positive and small sized association with optimism and patience $(\beta=0.54, \operatorname{Se} \beta=0.26, p=.039$, $\eta_{\mathrm{p}}^{2}=.03,95 \% \mathrm{CI}=.00-.09$ ), and (3) a significant negative and small sized association with negative attitudes toward suicide prevention $\left(\beta=-1.64, \mathrm{Se} \beta=0.65, p=.012, \mathrm{\eta}_{\mathrm{p}}^{2}=.04,95 \% \mathrm{CI}=.00\right.$ .11). The latter findings can be interpreted as a positive association between SCAF total scores 
and positive attitudes toward suicide prevention (i.e., the sign can be flipped for a negative association with a variable where higher scores are negative in connotation)

SCAF exploratory mediation pathways. In light of the robust association of the SCAF total score with three domains of self-injury/suicide prevention outcome expectations, and as informed by social-cognitive theory (Bandura, 2004; Burnette, 2015), we explored potential mediators that may explain SCAF-suicide/self-injury prevention attitude pathways. Specifically, we examined a multiple mediator model consisting of burnout subscales and the job satisfaction total score (i.e., five total mediating pathways) because these factors displayed largely meaningful associations with either or both the SCAF total score or suicide/self-injury prevention-related attitudes (see ESM for summary of total direct and indirect effects). Likewise, we included as covariates the demographics demonstrating significant multivariate associations with either job-related attitudes or suicide/self-injury prevention outcomes. Referencing H7 results and ESM Table 4, participant sex and knowing someone who died by suicide were therefore included in mediation models as covariates. A total of three mediation models were run, one for each significant multivariate association between the SCAF total score and respective suicide/self-injury prevention attitude domains. This yielded a mediation test for perceived ability to help self-harming patients, optimism and patience, and negative attitudes toward suicide prevention (the reader is referred to the ESM data analysis section for further model details).

The set of mediators displayed a significant total indirect effect in identifying pathways from the SCAF total score to perceived ability to help self-harming patients, $\beta=0.05$, se $\beta=0.02$, $95 \% \mathrm{BCa} \mathrm{CI}=.01-.09$. Of the five individual pathways, only job satisfaction was a significant (and positive with each link) pathway between the SCAF total score and perceived ability to help 
self-harming patients, $\beta=0.03$, se $\beta=0.02,95 \% \mathrm{BCa} \mathrm{CI}=.01$ to .07 (see ESM for summary of supplementary mediation model information). The set of mediators did not display a significant total indirect effect identifying pathways from the SCAF total score to optimism and patience, $\beta=0.05, \mathrm{se} \beta=0.03,95 \% \mathrm{BCa} \mathrm{CI}=-.01$ to. 11 . The set of mediators also did not display a significant total indirect effect identifying pathways from the SCAF total score to negative attitudes toward suicide prevention, $\beta=-0.06$, $\mathrm{se} \beta=0.04,95 \% \mathrm{BCa} \mathrm{CI}=-.15$ to. 01 .

\section{Discussion}

In the present investigation, we evaluated the psychometric properties of the SCAF. The following important advancements are noteworthy: (1) statistical support for a single-factor conceptualization and robust internal consistency; (2) notably low total scores for nursing assistants; (3) meaningful convergent associations with prior suicide prevention training, job satisfaction and enthusiasm; and (4) meaningful convergent and incremental validity associations with negative attitudes toward suicide prevention, perceived capacity to help self-harming patients, as well as optimism and patience working with self-harming patients. Offering important insight into the potential impacts of perceived suicide prevention skills as measured by the SCAF, we identified a social-cognitive theory (Bandura, 2004; Burnette et al., 2015) grounded pathway in which job satisfaction mediates the association between suicide competency/perceived self-efficacy (i.e., SCAF total score) and perceived ability to help selfharming patients (i.e., outcome expectations as defined by anticipated outcomes for the patient, effort to be exerted working with patients, and extent to which prevention efforts will have patient benefit; Gibb et al., 2010). Overall, our results provide support for the use of the SCAF in research, training, and prevention practice in psychiatric settings. 
Cramer and colleagues' (2013) review identified core suicide prevention skills focusing on the provider (e.g. managing one's reactions to suicide) and working with suicidal patients (e.g., knowing risk and protective factors). Present internal consistency and factor-analytic findings suggest these two broader themes can be conceptualized and measured under one umbrella of suicide prevention skills. Such a single-factor approach is consistent with how the SCAF has been used in student training (Cramer et al., 2016; Cramer \& Long, in press), and clinical and community health professions training (Cramer et al., 2017; La Guardia et al., in press). Given consistency in reliability, and meaningful pre-post training gains, it follows that suicide prevention practice may treat competency development as one overarching topic. This may be accomplished in psychiatric care settings through use of the SCAF in behavioral health supervision, self-reflective process/practice, and expanded training. Indeed, such efforts that include content such as clinical documentation and evidence-based interventions would answer recent calls in the UK behavioral health staff literature (Anewat et al., 2017), suggesting such advancements.

Contrary to prior literature (e.g., Botega et al., 2005; Saunders et al., 2012) suggesting nursing staff hold notably positive suicide-related attitudes, our findings suggest nursing assistants were lower in perceived skills. Interestingly, their mean total scores were similar to those reported in prior literature by behavioral health staff for counseling center staff and psychology trainees (Cramer et al., 2017), as well as an interdisciplinary group of community health providers (La Guardia et al., 2017), prior to exposure to a SCAF-based suicide prevention training. Given that other groups have responded well to SCAF-based training, and we found a modest positive association between SCAF scores and amount of prior suicide prevention 
training, it is possible that NHS nursing assistants, among other staff surveyed in the present study, may uniquely benefit from similar training.

Correlation patterns of SCAF scores with both positive job outcomes and suicide prevention attitudes/intentions are consistent with those of other suicide competency domains. For example, Gibb and colleagues (2010) demonstrated that emotional exhaustion and low personal attainment were job-related outcomes associated with perceived ability or intention to work with self-harming patients. SCAF correlates, however, are all positive in nature; that is, SCAF total scores demonstrated associations with positive job- and suicide prevention-specific correlates. Negative outcomes (e.g., psychological exhaustion) were unrelated. Moreover, consistent with a social-cognitive approach (Bandura, 2004; Burnette et al., 2015) incremental validity analyses point to the SCAF's potential impact on psychiatric staff anticipated suicide and self-injury outcomes for their patients. Moreover, mediation findings identified a positive pathway from high perceived skills to perceived ability to positively impact self-harming patients through positive job satisfaction. We know the high risk inpatient and community environments faced by behavioral health staff is potentially harmful to one's personal and professional wellbeing (Brems \& Johnson, 2009; Gibb et al., 2010). Yet, our findings collectively appear to suggest that those with high perceived mastery of skills needed to handle the challenging behavioral health setting enjoy their job more and, in turn, feel a stronger sense of ability to help. Such a pattern also raises the possibility that enhancing suicide prevention competency can buffer against any potential negative job-related (e.g., burnout) influences in relation to job satisfaction or confidence in helping high risk patients. In sum, building SCAF skills may potentially affect clinical acumen for high risk patients and behavioral health staff job satisfaction and retention. 
The present study represents a valuable step forward in establishing a psychometrically sound measure of suicide prevention competencies. Given the psychometric support for the SCAF in this study, it appears appropriate for quantifying provider self-perceived suicide prevention skills in a variety of contexts. For instance, prior studies (e.g., Cramer et al., 2016; La Guardia et al. in press) have demonstrated the SCAF total score is a meaningful training outcome sensitive to positive change among a variety of health professionals. Especially in light of prior research showing negative suicide prevention attitudes among inpatient and other healthcare staff (e.g., Herron et al., 2001), we recommend continued SCAF utilization in suicide prevention training. Additionally, where healthcare organizations are assessing current capacity and gaps to respond to patient mental health concerns, the SCAF may serve as a critical tool for such needs assessments. SCAF and similar instruments (e.g., Hung et al., 2012) are intended as self- and observer-rated forms. As such, individual or group clinical supervision offers an appropriate setting in which completion of the SCAF by supervisee and supervisor can yield identification of clinician strengths and areas of growth. Prospective use of the SCAF in supervision, training, and related practices is worthy of future attention.

Despite promising SCAF findings, this study possesses limitations that provide avenues for future research. A few expected correlation patterns failed to reach significance or were smaller than expected. The low SBI guilt (Gil-Monte \& Manzano-Garcia, 2015) and ASHP (Gibb et al., 2010) subscale internal consistency values may explicate these non-significant findings. In the instance of the SBI, prior literature (Gil-Monte \& Olivares, 2011; Gil-Monte \& Mazano-Garcia, 2015) established psychometric properties in non-English speaking populations, and yet provided an English-translated version of the scale in the literature. Item content in the Guilt subscale may have translated or been interpreted differently across cultures, explaining the 
low internal consistency. Thus, our results necessitate caution regarding construct validation correlates of the SCAF, and future research should extend to other suicide prevention-focused (e.g., suicide prevention literacy and suicide stigma; Batterham et al., 2013) and well-being measures. Moreover, our cross-sectional design and sample size precluded test-retest, inter-rater and confirmatory factor analyses. Future SCAF psychometric work should address these gaps through prospective designs and larger samples of healthcare professionals such as clinical trainees or community healthcare professionals (i.e., those populations utilized in previous SCAF-based training literature). Given the potential use of the SCAF in clinical supervision, restriction of SCAF evaluation to the self-report format in our study raises next logical steps of examining SCAF inter-rater agreement through comparison to expert raters of suicide prevention skills, as well as associations with other provider characteristics and patient outcomes. Finally, several studies (Cramer et al., 2016, 2017; La Guardia et al., in press) have established positive impacts of SCAF-based trainings on a range of suicide prevention competencies and healthcare professions disciplines. Our findings provide empirical rationale to examine both longitudinal effects of such training on SCAF scores, as well as extension of training impacts to behavioral health staff job satisfaction and retention. 


\section{References}

Ahmedani, B.K., Simon, G.E., Stewart, C., Beck, A., Waitzfelder, B.E., Rossom, R., ...

Operskalski, B. H. (2014). Health care contacts in the year before suicide death. Journal of General Internal Medicine, 29, 870-877.

Arensman, E. (2017). Assessing the effectiveness of suicide prevention programmes: Integrating outcome and process evaluation. In D. De Leo, V. Poštuvan, D. De Leo, V. Poštuvan (Eds.). Resources for suicide prevention: Bridging research and practice (pp. 57-63). Boston, MA, US: Hogrefe Publishing.

Awenat, Y., Peters, S., Shaw-Nunez, E., Gooding, P., Pratt, D., \& Haddock, G. (2017). Staff experiences and perceptions of working with in-patients who are suicidal: qualitative analysis. British Journal of Psychiatry, 211, 103-108.

Bandura, A. (1997). Self-efficacy: the exercise of control. New York, NY: W.H. Freeman and Company.

Bandura, A. (2004). Health promotion by social cognitive means. Health Education \& Behavior, $31,143-164$.

Batterham, P.J., Calear, A.L., \& Christensen, H. (2013). Correlates of suicide stigma and suicide literacy in the community. Suicide and Life-Threatening Behavior, 43, 406-417.

Botega, N.J., Reginato, D.G., da Silva, S.V., da Silva Cais, C.F., Rapeli, C.B., Mauro, M.L.F., \& ...Stefanello, F. (2005). Nursing personnel attitudes toward suicide: the development of a measure. Brazilian Review of Psychiatry, 27(4), 315-318.

Botega, N., Silva, S., Reginato, D., Rapeli, C., Cais, C., Mauro, M., \& ... Cecconi, J. (2007). Maintained attitudinal changes in nursing personnel after a brief training on suicide prevention. Suicide and Life-Threatening Behavior, 37, 145-153. 
Brems, C., \& Johnson, M.E. (2009). Self-care in the context of threats of violence or self-harm from clients. In J. J. Werth, E.R. Welfel, G.H. Benjamin, J.J. Werth, E.R. Welfel, G.H. Benjamin (Eds.). The duty to protect: Ethical, legal, and professional considerations for mental health professionals (pp. 211-227). Washington, DC, US: American Psychological Association.

Brodsky, B.S., Spruch-Feiner, A., \& Stanley, B. (2018). The Zero Suicide Model: Applying evidence-based suicide prevention practices to clinical care. Frontiers in Psychiatry, 9, 33.

Burnette, C., Ramchand, R., \& Ayer, L. (2015). Gatekeeper training for suicide prevention: A theoretical model and review of empirical literature. Rand Health Quarterly, 5, 16.

Cerel, J., Brown, M.M., Maple, M., Singleton, M., Venne, J., Moore, M., \& Flaherty, C. (2018). How many people are exposed to suicide? Not six. Suicide and Life-Threatening Behavior.

Cramer, R.J., Bryson, C.N., Eichorst, M.K., Keyes, L.N., \& Ridge, B.E. (2017). Conceptualization and pilot testing of a core competency-based training workshop in suicide risk assessment and management: Notes from the field. Journal of Clinical Psychology, 73, 233-238.

Cramer, R.J., Bryson, C.N., Stroud, C.H., \& Ridge, B.E. (2016). A pilot test of a graduate course in suicide theory, risk assessment, and management. Teaching of Psychology, 43, $238-242$.

Cramer, R.J., Johnson, S.M., McLaughlin, J., Rausch, E.M., \& Conroy, M.A. (2013). Suicide risk assessment training for psychology doctoral programs: Core competencies and framework for training. Training and Education in Professional Psychology, 7(1), 1-11. 
Cramer, R.J., \& Long, M.M. (in press). Competency-based suicide prevention education: Implementation of a pilot course for undergraduate health professions students. Academic Psychiatry.

Cramer, R.J., \& Kapusta, N.D. (2017). A social-ecological framework of theory, assessment and prevention of suicide. Frontiers in Psychology, 8, 1756.

Crichton, J.H.M. (2009). Defining high, medium, and low security in forensic mental healthcare: the development of the Matrix of Security in Scotland. Journal of Forensic Psychiatry \& Psychology, 20, 333-353.

Ferguson, M.S., Reis, J.A., Rabbetts, L., Ashby, H., Bayes, M., McCracken, T., \& ... Procter, N. G. (2018). The effectiveness of suicide prevention education programs for nurses: A systematic review. Crisis, 39, 96-109.

Fleury, M., Grenier, G., \& Bamvita, J. (2017). Job satisfaction among mental healthcare professionals: The respective contributions of professional characteristics, team attributes, team processes, and team emergent states. Sage Open Medicine.

Herron, J., Ticehurst, H., Appleby, L., Perry, A., \& Cordingley, L. (2001). Attitudes toward suicide prevention in front-line health staff. Suicide and Life-Threatening Behavior, $31(3), 342-347$.

Gibb, S.J., Beautrais, A.L., \& Surgenor, L.J. (2010). Health-care staff attitudes towards self-harm patients. Australian and New Zealand Journal of Psychiatry, 44, 713-720.

Gil-Monte, P.R., \& Manzano-Garcia, G. (2015). Psychometric properties of the Spanish Burnout Inventory among staff nurses. Journal of Psychiatric and Mental Health Nursing, 22, 756-763. 
Gil-Monte, P.R., \& Olivares, V. (2011). Psychometric properties of the 'Spanish Burnout Inventory' in Chilean professionals working to physical disabled people. The Spanish Journal of Psychology, 14, 441-451.

Jahn, D.R., Quinnett, P., \& Ries, R. (2016). The influence of training and experience on mental health practitioners' comfort working with suicidal individuals. Professional Psychology: Research and Practice, 47, 130-138.

Jiao, Y., Phillips, M.R., Sheng, Y., Wu, G., Li, X., Xiong, W., \& Wang, L. (2014). Crosssectional study of attitudes about suicide among psychiatrists in Shanghai. BMC Psychiatry, 14, 87.

La Guardia, A.C., Cramer, R.J., Brubaker, M., \& Long, M.M. (in press). Community mental health provider responses to a competency-based training in suicide risk assessment and prevention. Community Mental Health Journal.

Long, M., Manktelow, R., \& Tracey, A. (2013). We are all in this together: Working towards a holistic understanding of self-harm. Journal of Psychiatric and Mental Health Nursing, $20,105-113$.

Luoma, J.B., Martin, C.E., \& Pearson, J.L. (2002). Contact with mental health and primary care providers before suicide: A review of the evidence. The American Journal of Psychiatry, 159, 909-916.

Mitchell, S.M., Brown, S.L., Roush, J.F., Bolaños, A.D., Littlefield, A.K., Marshall, A.J., \& ... Cukrowicz, K.C. (2017). The clinical application of suicide risk assessment: A theorydriven approach. Clinical Psychology \& Psychotherapy, 24(6), 1406-1420.

Morse, G., Salyers, M.P., Rollins, A.L., Monroe-DeVita, M., \& Pfahler, C. (2012). Burnout in mental health services: A review of the problem and its remediation. Administration and 
Policy in Mental Health and Mental Health Services Research, 39, 341-352.

Office for National Statistics. (2018). Suicide in the UK: 2017 registrations. Retrieved from: https://www.ons.gov.uk/peoplepopulationandcommunity/birthsdeathsandmarriages/deat s/bulletins/suicidesintheunitedkingdom/2017registrations

Roush, J.F., Brown, S.L., Jahn, D.R., Mitchell, S.M., Taylor, N.J., Quinnett, P., \& Ries, R. (2018). Mental health professionals' suicide risk assessment and management practices: The impact of fear of suicide-related outcomes and comfort working with suicidal individuals. Crisis, 39, 55-64.

Rudd, M.D. (2006). Assessment and management of suicidality. Sarasota, FL: Professional Resource Press.

Samaritans. (2017). Suicide statistics report 2017 (including data for 2013-2015). Retrieved: https://www.samaritans.org/sites/default/files/kcfinder/files/Suicide_statistics_report_20 7_Final.pdf

Saunders, K.A., Hawton, K., Fortune, S., \& Farrell, S. (2012). Attitudes and knowledge of clinical staff regarding people who self-harm: A systematic review. Journal of Affective Disorders, 139, 205-216.

Schmitz, W.J., Allen, M.H., Feldman, B.N., Gutin, N.J., Jahn, D.R., Kleespies, P.M., \& ... Simpson, S. (2012). Preventing suicide through improved training in suicide risk assessment and care: An American Association of Suicidology Task Force report addressing serious gaps in U.S. mental health training. Suicide and Life-Threatening Behavior, 42, 292-304. 
Shepard, D.S., Gurewich, D., Lwin, A.K., Reed, G.A., \& Silverman, M.M. (2016). Suicide and suicidal attempts in the United States: Costs and policy implications. Suicide and Life Threatening Behavior, 46, 352-362.

Silva, C., Smith, A.R., Dodd, D.R., Covington, D.W., \& Joiner, T.E. (2016). Suicide-related knowledge and confidence among behavioral health care staff in seven states. Psychiatric Services, 67, 1240-1245.

World Health Organization. (2015). Suicide. Retrieved from: http://www.who.int/mediacentre/factsheets/fs398/en 